\title{
Design of Printed Monopole ANTENNA For MICROWAVE COMMUNICATION
}

\author{
Yogesh B. Thakare ${ }^{1}$ \\ ${ }^{1}$ Department of Electronics and Telecommunication Engineering, \\ PVG's COET (Affiliated to University of Pune), Pune-09 (INDIA).
}

\begin{abstract}
A printed monopole in its planar and vertical configuration has been designed, fabricated and analyzed for microwave applications on low cost FR4 substrate material of thickness, $h=1.56 \mathrm{~mm}$ and relative permittivity, $\varepsilon_{r}=4.3$. The designed planar monopole has been simulated and experimented to find its frequency response with coplanar waveguide feed to exhibit dual band characteristics with $-10 \mathrm{~dB}$ reflection loss bandwidth of $45.078 \%$ (i.e. 1.5:1 between 1.334 and 2.109 GHz) and 114.92 \% (i.e. 3.7: 1 between 3.99 and $14.77 \mathrm{GHz}$ ). The vertical monopole using the same patch has also been simulated and experimented and $-10 \mathrm{~dB}$ reflection loss bandwidth of $173.67 \%$ (14.2:1 between 0.925 and $13.125 \mathrm{GHz})$ has been obtained. The antenna finds many applications in microwave bands.
\end{abstract}

\section{KEYWORDS}

Antenna, Fractal, Monopole, Microwave, UWB.

\section{INTRODUCTION}

Now days, there is a great demand for wide bandwidth in microwave frequency region due to increasing number of wireless applications and to boost high data rate for faster communication [1]-[5]. The conventional configurations of microstrip antenna (MSA) offering narrow impedance bandwidth are capable to satisfy necessary bandwidth due their resonant nature [1]. So, wider bandwidth [6] in an antenna has become a challenge. Many techniques such as stacked patches, multi-resonator configurations using excited and parasitic patches and gap coupling or proximity coupling method, monopole geometry, slot loading using microstripline feed, fractal grounding [1]-[25] have been proposed. In this context, D. M. Pozar has stated that the permittivity, $\varepsilon_{\mathrm{r}}$ and thickness, h of the dielectric substrate of the patch antenna influences its bandwidth [1]-[4] and decrease in $\varepsilon_{\mathrm{r}}$ and increase in the thickness of the substrate increases the bandwidth. But, this technique increases size, weight, and cost of an antenna [4]. With thick substrate and low dielectric constant, bandwidth of 5 to $15 \%$ can be achieved in MSA. Any further increase in the substrate thickness to increase bandwidth decreases the efficiency and increases cross-polar level and inductive reactance due to increased probe height. Further, this leads to excitation of substrate modes at discontinuities to generate scatter at other discontinuities to offer undesirable radiations at undesirable polarization. In microstrip arrays, this leads to serious performance degradation. The antenna efficiency, polarization, and radiation patterns are degraded due to excitation of higher order modes of the patch that contribute to the radiation. This problem can be solved by using a patch without the substrate with a perpendicular ground plane fed by a coaxial feed known as vertical monopole.

In monopole, the patch is fed along the periphery which increases the height keeping effective dielectric constant of one to yield a large bandwidth. If height of the monopole is very large, the bottom ground plane would have negligible effect, and can be removed. Such configuration is 
International Journal Of Microwave Engineering (JMICRO) Vol.1, No.4, October 2016

known as planar monopole which can obtain broadband impedance characteristics [24]-[25]. In monopole, coplanar waveguide (CPW) is popular since it can be printed on same side of patch on the substrate and offer good radiation characteristics at low cost.

The planar and vertical monopole configurations are very popular to satisfy the growing bandwidth requirements of various wireless communication standards [1]-[25]. This includes cell phone antenna $(0.8-11 \mathrm{GHz})$, DTV $(0.470-0.860 \mathrm{GHz})$, GPS (1.565-1.585 \& 1.227-1.575GHz), PCS (1.8-2GHz), UMTS (2-2.3GHz), WiMax-WiFi-Wibro-other OFDM (2.3 - $3.7 \mathrm{GHz})$, Bluetooth (2.4-2.4835GHz), WLAN (2.4-2.4835, 5.15-5.35, and 5.725-5.850GHz), Low band UWB (3.1-5.15GHz), GSM900(890-960MHz), GSM1800, DCS1800 (1.710-1.880GHz), IMT2000 (1.885-2.2GHz), ISM 2.4/5.2/5.8 (2.4-2.484 / 5.725-5.825 / 5.15-5.35GHz), UWB (3.1-10.6 GHz), AMPC800, WCDMA, UMTS2000 (3G)/ IMT2000 (1.885-2.200GHz), UMTS2000 (1.920-2.170GHz), etc [1]-[25]. Out of all the above-mentioned wireless systems, ultra-wide bandwidth (UWB) (BW-7.5GHz between $3.1-10.6 \mathrm{GHz}$ ) wireless technology is most sought for very high-data-rate and short-range wireless communication systems, security systems, rejection of multipath effect, modern radar systems, etc. A universal antenna at least demands five cellular bands (GSM850/900/1800/1900/3G), Wireless LAN, Bluetooth, Digital TV (DVB-H), FM radio and GPS. In near future, several new wireless systems such as RFID, UWB, and WiMAX etc. may be integrated to the terminal. To fulfill above demands for bandwidth in one antenna will necessarily require compact wide, broad band, UWB or SWB microwave antenna. This wide bandwidth requirement can be achieved using compact printed monopole antennas [1]-[4]. Monopoles with circular, elliptical, square (rectangular), bow-tie, diamond, and trapezoidal sheets, fractals have been designed and investigated [1]-[26].

Fractal is known as fractus in Latin language to mean "broken" or "fractured" geometry. "A fractal is a rough or fragmented geometric shape that can be splitted into parts, each of which is (at least approximately) a reduced-size copy of the whole" [19]. Around 1975, B. Mandelbrot has reported existence of non-Euclidean geometries called as fractal and its use in many natural findings. A fractal is an integral part of nature and has applications in antennas for multiband wireless communication. The self similarity and space filling property in fractal can achieve multiband and miniaturization characteristics in antennas [19]-[24]. Several fractal antennas have been reported for compact, multiband and broadband applications [1]-[4], [8]-[25].

This work presents the design and analysis of a printed fractal antenna in its planar and monopole [11],[12],[16] configuration fabricated on FR-4 (an international grade designation for fiberglass reinforced epoxy laminates that are Flame Retardant or Resistant) substrate suitable for wideband applications.

\section{DESIGN OF THE PRINTED MONOPOLE ANTENNA}

The printed monopole antenna has been designed on low cost FR4 (glass substrate $\left(\varepsilon_{\mathrm{r}}=4.3\right.$, $\mathrm{h}=1.56 \mathrm{~mm}$ ) with patch size of $121 \mathrm{~cm}^{2}$ area excluding the CPW feed area. As shown in Figure1, a fractal patch has been designed and this fractal patch serves as initiator (Figure1e) in the design of the printed monopole antenna as shown in Figure2. The design of initiator has been presented in Fig. 1a-e using following steps.

1. An equilateral triangular slot of dimension $66 \mathrm{~mm}$ with its vertex at $0^{0}$ is generated in the conventional patch (Figure1b),

2. Using patch generated in design step 1, a second equilateral triangular slot of same dimension with its vertex at $90^{\circ}$ is generated (Figure1c),

3. Using patch generated in design step 2, a third equilateral triangular slot of same dimension with its vertex at $180^{\circ}$ is generated (Figure1d), 
International Journal Of Microwave Engineering (JMICRO) Vol.1, No.4, October 2016 4. Lastly, using patch generated in design step 3, a fourth equilateral triangular slot of same dimension with its vertex at $270^{\circ}$ is generated (Figure1e).
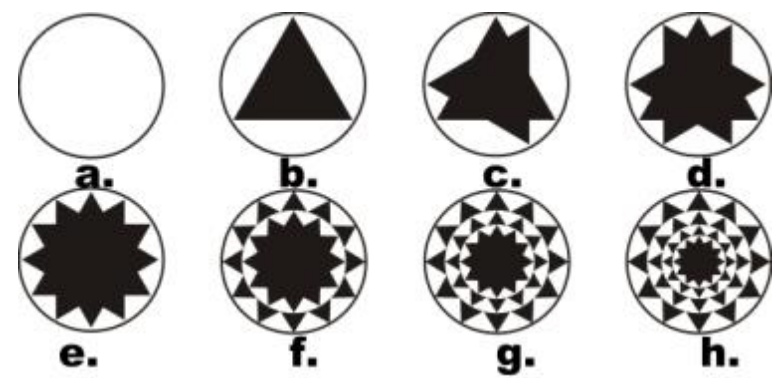

Figure 1. Design of iterative fractal patch [11-12], [26].

The scale down copy of fractal initiator is added upto four iterations inside the initiator in Figure1e in steps as shown in Figure1f, $1 \mathrm{~g}$ and $1 \mathrm{~h}$ to design final fractal patch used in monopole. The design dimensions of the printed fractal patch are shown in Figure2. Using this printed fractal patch, $50 \Omega \mathrm{CPW}$ fed monopole is designed in planar and vertical configuration as shown in Figure 3 and 4 respectively. The photographs of the fabricated monopole on low cost FR4 substrate $\left(\varepsilon_{\mathrm{r}}=4.3, \mathrm{~h}=1.56 \mathrm{~mm}\right)$ in both planar and vertical configurations are shown in Figure5.

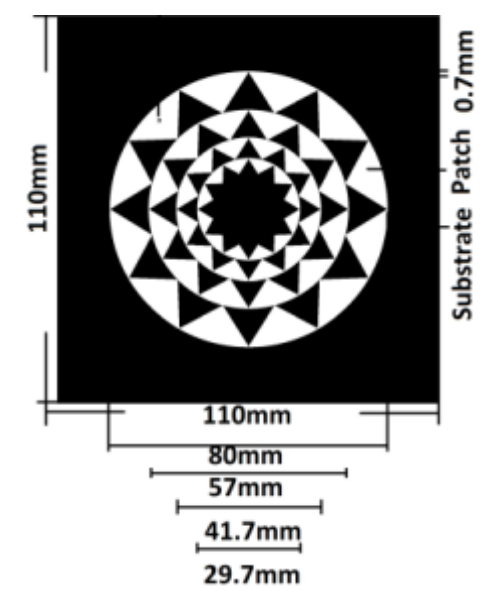

Figure 2. Iterative fractal patch design dimensions [16].
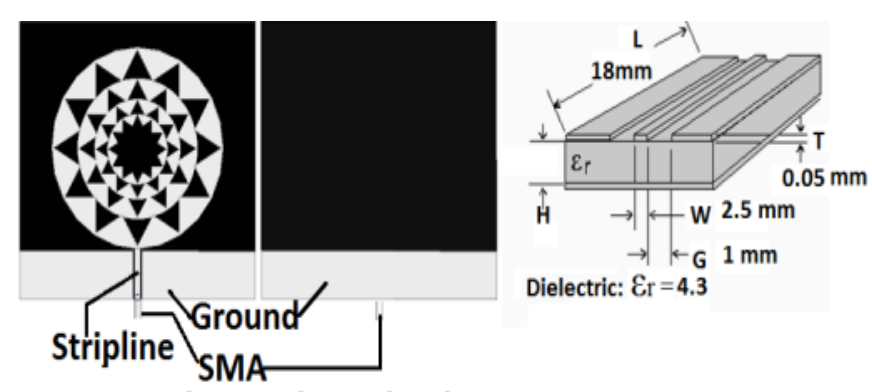

Dielectric: $\mathrm{Er}=4.3$

a. Top side

b. Back side C. CPW feed with ground plane

Figure 3. Printed monopole in planar configuration [16]. 
International Journal Of Microwave Engineering (JMICRO) Vol.1, No.4, October 2016

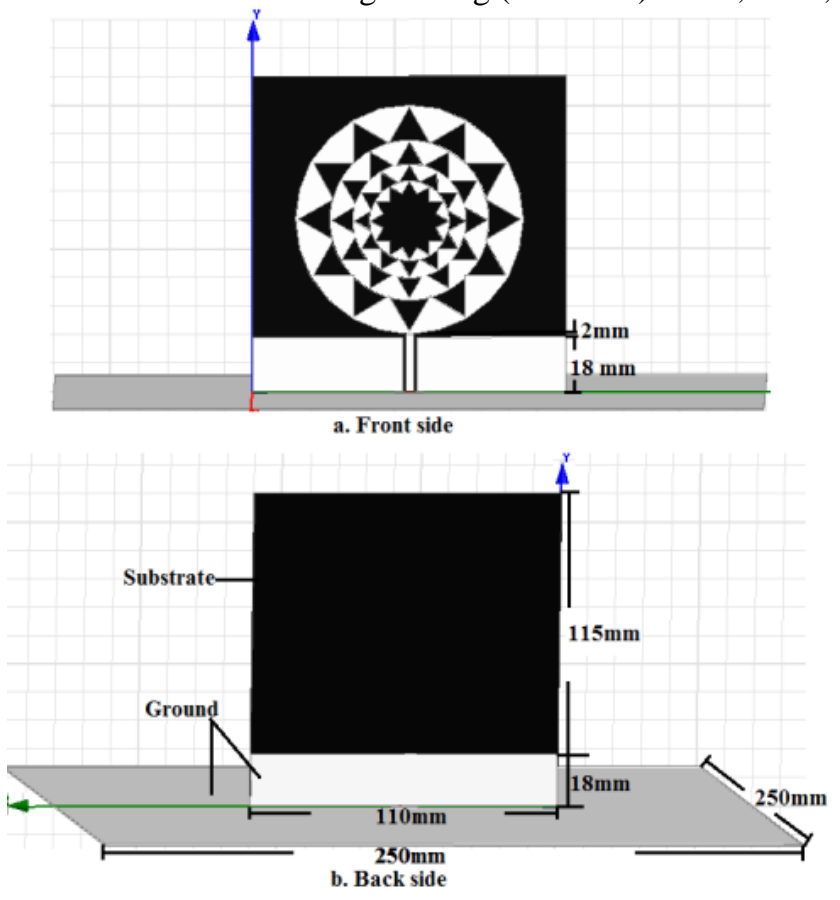

Figure 4. Printed monopole in vertical configuration [16].

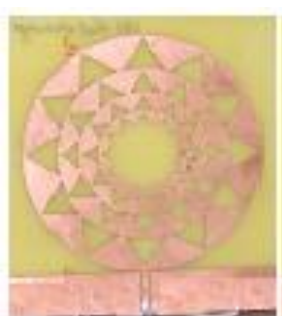

a. Front View

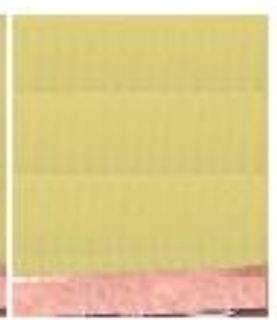

b. Rear View

i .Planar configuration

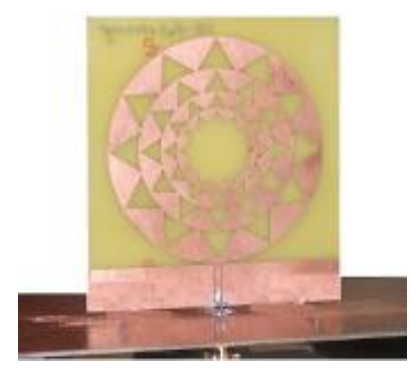

ii. Vertical configuration

Figure 5. Photographs of designed monopole antenna.

\section{RESULTS AND DISCUSSIONS}

The reflection loss plots of planar and vertical configurations of the designed monopole are simulated and measured as shown in Figure6 and 7 respectively and compared. 


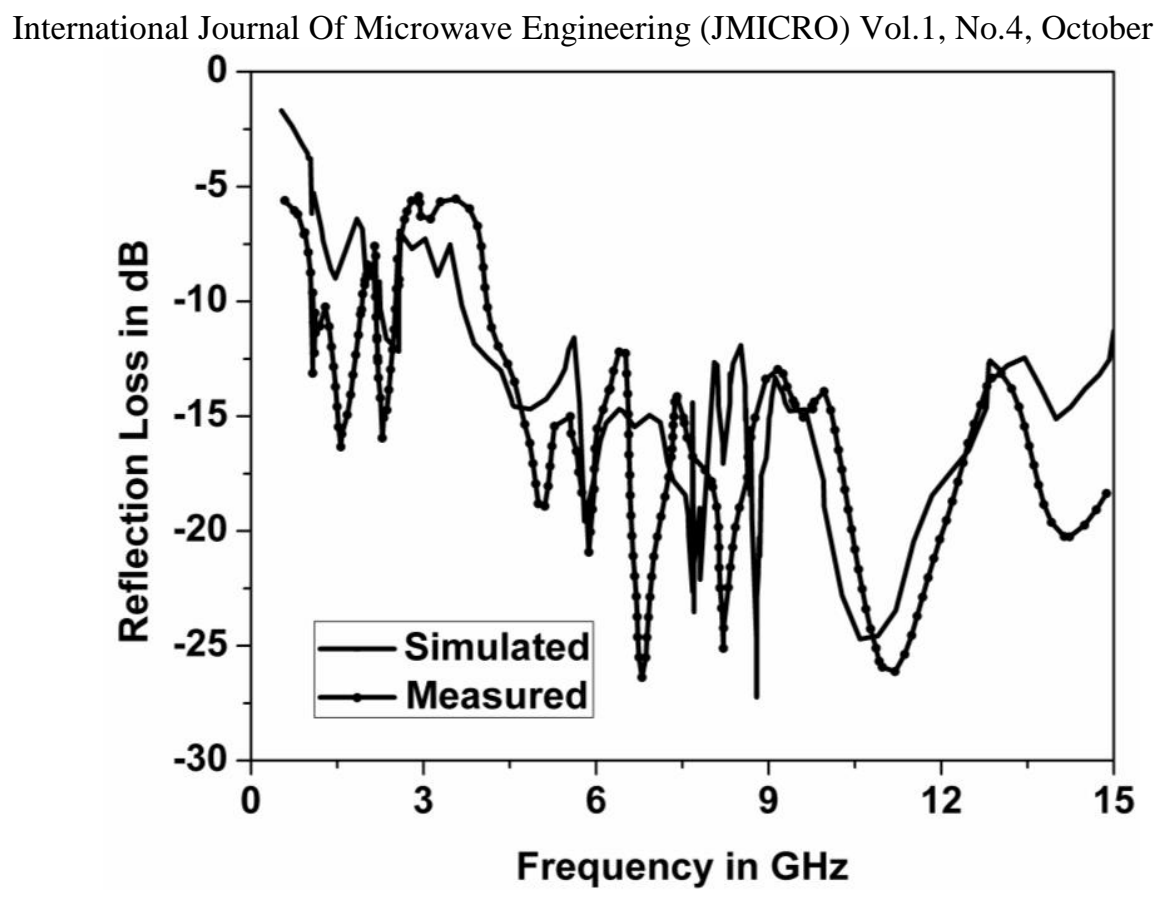

Figure 6. The reflection loss plots of printed planar monopole.

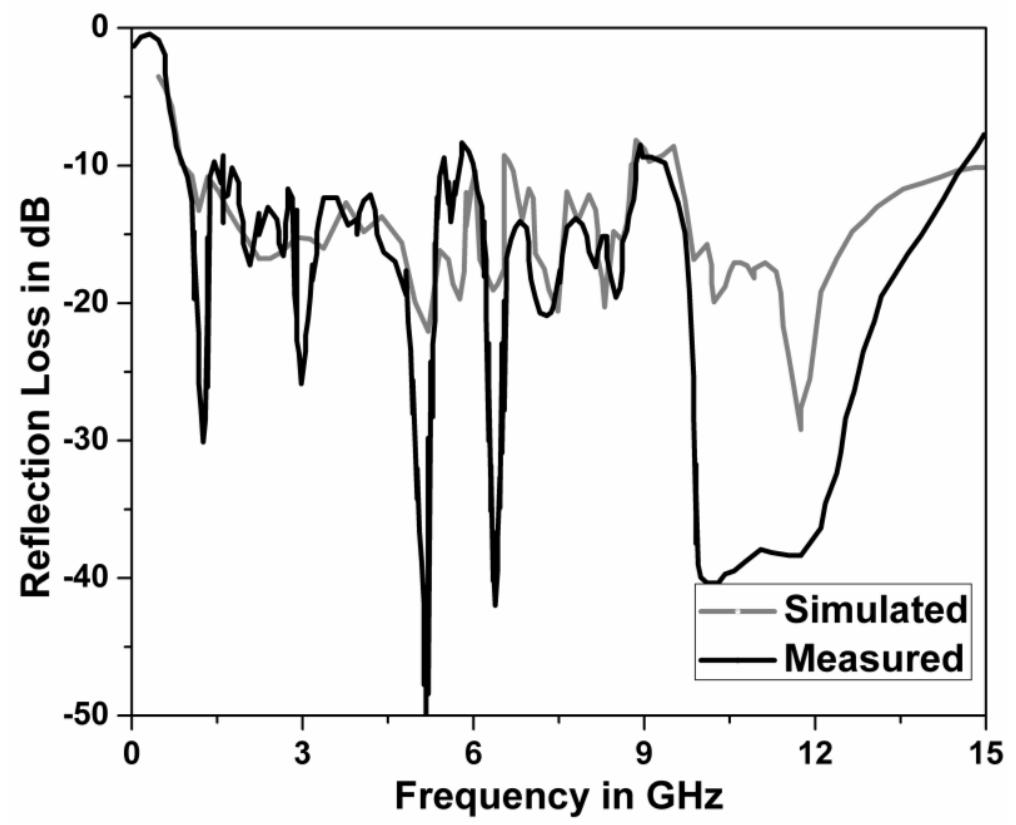

Figure 7. Reflection loss plot of printed vertical monopole.

The simulated and measured results are in close agreement as seen in Figure 6 and 7. From Figure6, the observed $-10 \mathrm{~dB}$ reflection loss bandwidth in planar monopole using designed iterative fractal patch is found to be $774.7489 \mathrm{MHz}$ for the frequency swept between 1334.2898 $\mathrm{MHz}$ and $2109.03873 \mathrm{MHz}$ whereas it is $10.78 \mathrm{GHz}$ for frequency swept between $3.99 \mathrm{GHz}$ to 14.77 GHz. The percentage fractional bandwidth with first band is $45.07854 \%$ whereas 114.92 $\%$ with second band. The index of breadth of $2^{\text {nd }}$ band or relative bandwidth, $\mu_{\mathrm{b}}=1.1492$ satisfies UWB requirement [23]. A quality factor, $Q$ of 0.6153 is calculated. The planar monopole antenna may be useful for unified applications covering GPS (1.565-1.585 and 1.227$1.575 \mathrm{GHz})$, PCS (1.8-2GHz), WLAN (5.15-5.35, and 5.725-5.850GHz), GSM1800, DCS1800 
As shown in Figure7, for vertical monopole using same iterative fractal patch, the reflection loss bandwidth of $12.2 \mathrm{GHz}$ for frequency swept between 0.925 to $13.125 \mathrm{GHz}$ is observed. The calculated percentage fractional bandwidth is found to be $173.66 \%$. This gives $\mu_{\mathrm{b}}=1.7366$ far greater than 1 as required in UWB communication. This is a super wideband (SWB) [11] response of the vertical monopole. The frequency ratio of 14.189 and $Q$ of 0.40717 have been calculated. It is found that increase in the bandwidth by monopole results into decrease in $\mathrm{Q}$ compared to planar monopole. The vertical monopole antenna may be useful for unified microwave applications covering GPS (1.565-1.585 and 1.227-1.575GHz), PCS (1.8 - 2GHz), UMTS (2-2.3GHz), WiMax-WiFi-Wibro-other OFDM (2.3-3.7GHz), Bluetooth (2.4$2.4835 \mathrm{GHz})$, WLAN $(2.4-2.4835,5.15$ to 5.35 , and $5.725-5.850 \mathrm{GHz})$, Low band UWB (3.1$5.15 \mathrm{GHz})$, GSM1800, DCS1800 (1.710-1.880 GHz), ISM 2.4/5.2/5.8 (2.4-2.484 / 5.725-5.825 / 5.15-5.35 GHz), UWB (3.1-10.6GHz), AMPC800, UMTS2000 (3G) / IMT2000 (1.8852.200GHz),UMTS2000 (1.920-2.170GHz) [37]-[39], etc.

The comparative study of planar and vertical configuration of monopole is shown in Figure8.

It has been found that planar monopole is a dual band antenna whereas vertical monopole is a single band antenna between $0.925-13.125 \mathrm{GHz}$ at the cost of large perpendicular ground plane to increase its overall dimensions compared to its planar configuration.

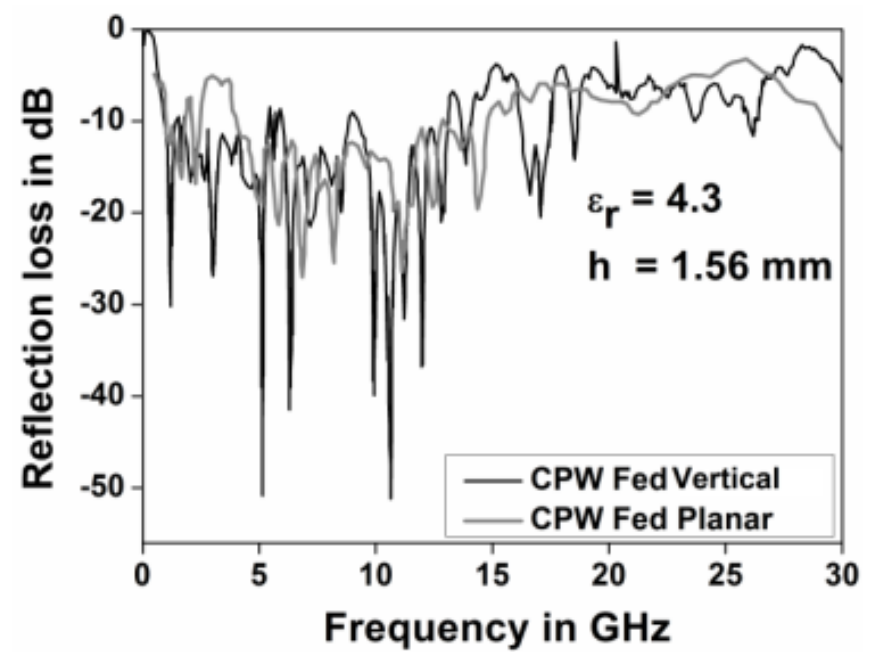

Figure 8. Comparative reflection loss plot of printed planar and vertical monopole.

In vertical monopole as seen in Figure8, the resonating frequency shifts towards lower frequency side at $0.925 \mathrm{GHz}$ compared to $1.334 \mathrm{GHz}$ and $3.99 \mathrm{GHz}$ in planar monopole. This is due to the increase in the volume of the vertical monopole compared to its planar configuration. Further, the unified percentage bandwidth enhancement in vertical monopole is greater than planar monopole using the same iterative fractal patch.

The increase in bandwidth for planar as well as vertical monopole is due to the coupled phenomenon among the fractal multi-resonant structures exciting closely spaced fundamental as well as higher order modes in monopole geometry when fed with CPW feed. This is similar to excitation of higher order modes in conventional patch monopole undergoing smaller impedance variation to offer large impedance bandwidth. Further, the monopole is circular in nature to its outer boundary. In circular monopole, the excited higher order modes are so closely spaced that they get mixed to give unified large bandwidth with CPW feed. This is called as coupled 
International Journal Of Microwave Engineering (JMICRO) Vol.1, No.4, October 2016

phenomenon of resonance. Also, increase in the bandwidth in the planar or vertical monopole may be explained as the cause of increase in the effective diameter similar to the thin vertical wire monopole over the ground plane, whose bandwidth increases with increase in its diameter. Further, like conventional planar monopole (which is viewed as a MSA on a very thick air substrate which offers large impedance bandwidth), planar fractal monopole also offers increase in bandwidth. The shape and size of the printed patches in these printed monopole antennas are the parameters of optimization to obtain very large impedance bandwidth for broadband monopole antenna.

As seen in Figure 9, the simulated peak and realized peak gain of the planar monopole antenna are almost varying equally to ensure maximum of $9 \mathrm{~dB}$ and minimum of $-18 \mathrm{~dB}$ gain in UWB band.

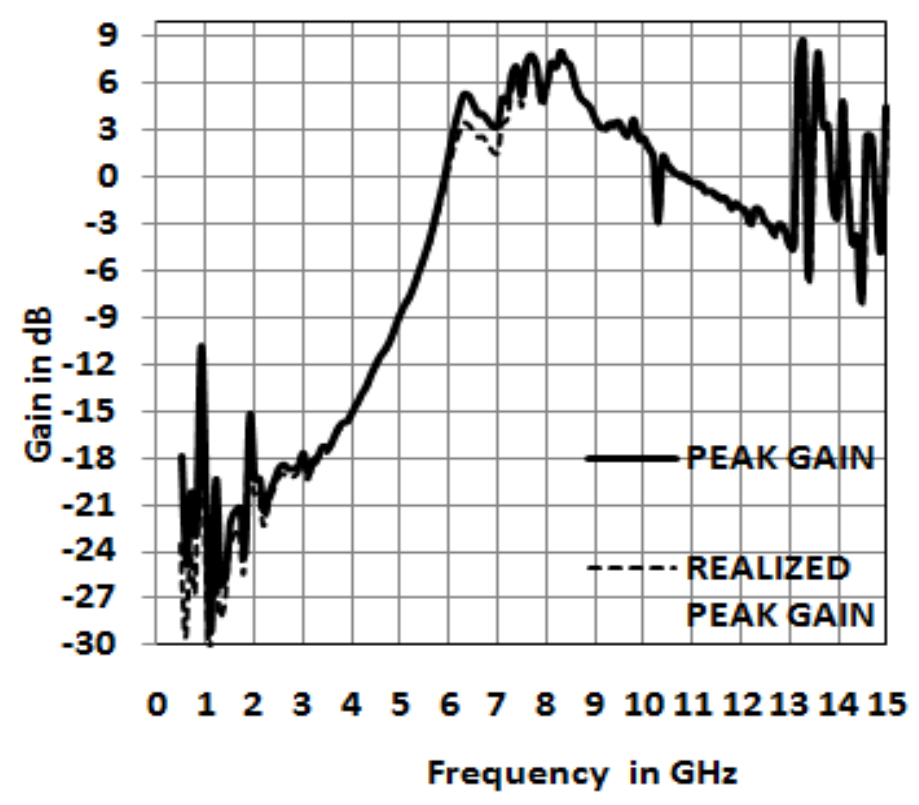

Figure 9. Gain vs. frequency plot of CPW fed planar monopole.

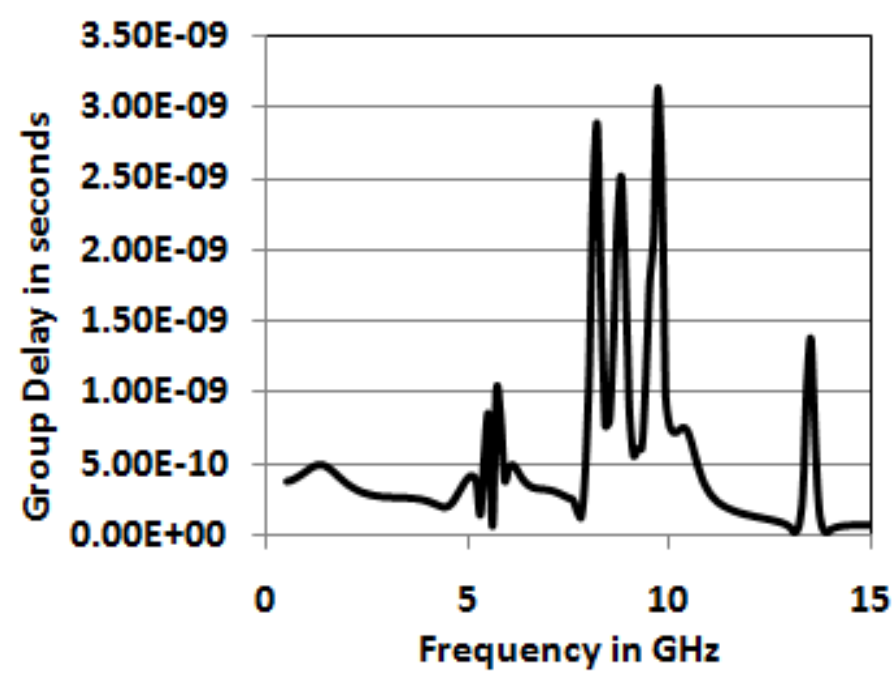

Figure 10. Group delay vs. frequency plot of CPW fed planar monopole. 
International Journal Of Microwave Engineering (JMICRO) Vol.1, No.4, October 2016

The small group delays of nano seconds in the planar monopole as shown in Figure10 has made the antenna usful in high speed data communication.

Radiation patterns: The CPW fed planar monopole is simulated and 2D radiation patterns and complex surface current distribution are plotted in Figure 11 at 4, 6, 8 and $10 \mathrm{GHz}$ respectively. The radiation patterns in Figure 11 and 12 are found similar at various frequencies.

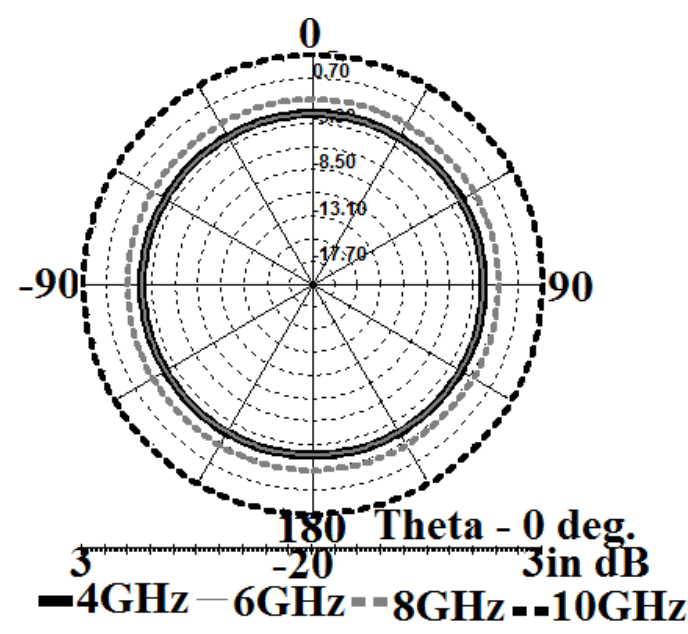

i.

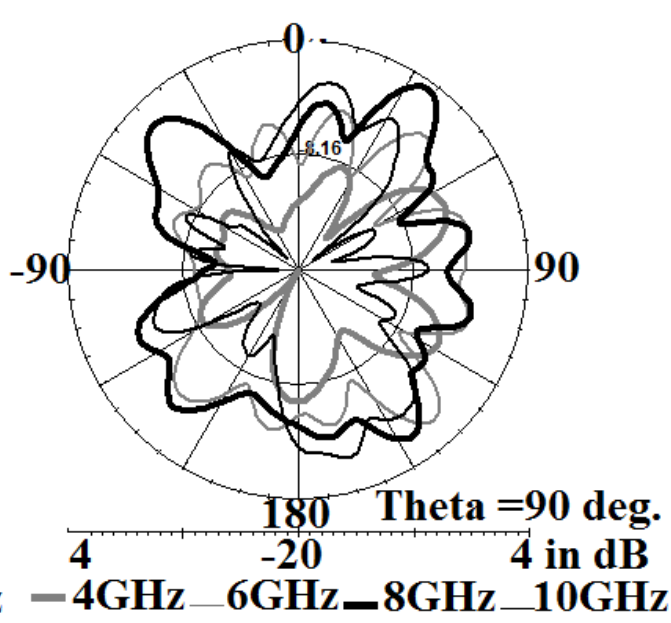

ii.

Figure 11. Radiation pattern of the CPW fed planar monopole at varying frequencies and theta 0 and 90 deg.

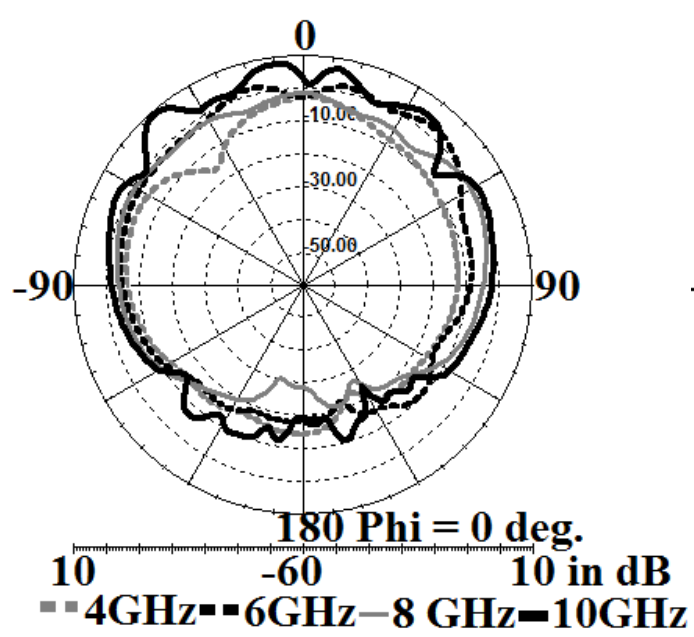

iii.

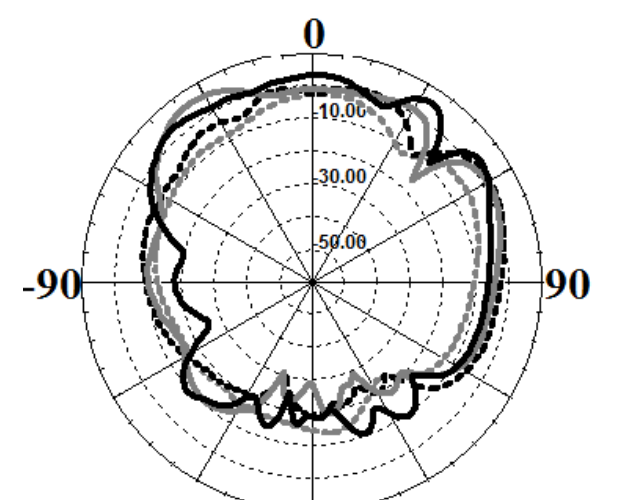

$180 \mathrm{Phi}=90 \mathrm{deg}$.

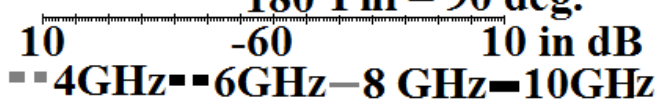

iv.

Figure 12. Radiation pattern of the CPW fed planar monopole at varying frequencies and phi 0 and 90 deg.

The simillarity in radiation patterns is due to self-similar current distribution on the antenna at these frequencies [24] as shown in Figure13. The radiation patterns at theta $=0 \mathrm{deg}$. is omnidirectional in phi plane at given frequencies but patterns get distorted at theta $=90 \mathrm{deg}$. in this plane as shown Figure11(i) and Figure11(ii). The maximum gain of $3 \mathrm{~dB}$ is obtained at 10 $\mathrm{GHz}$ in this phi plane. The radiation patterns are nearly omnidirectional at phi $=0$. 

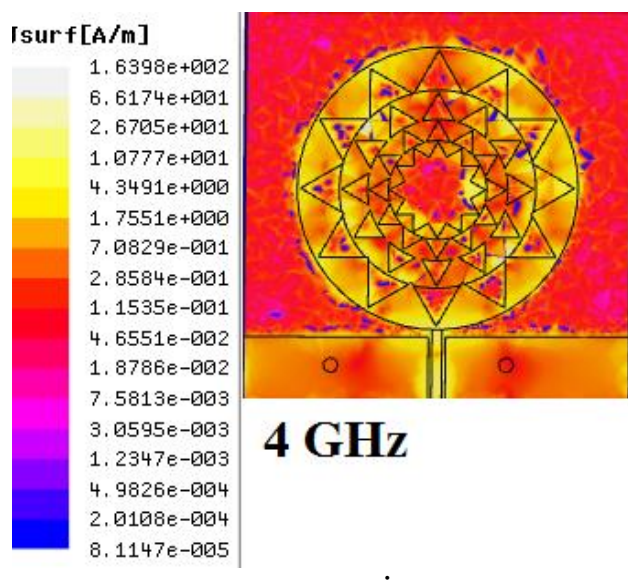

i.
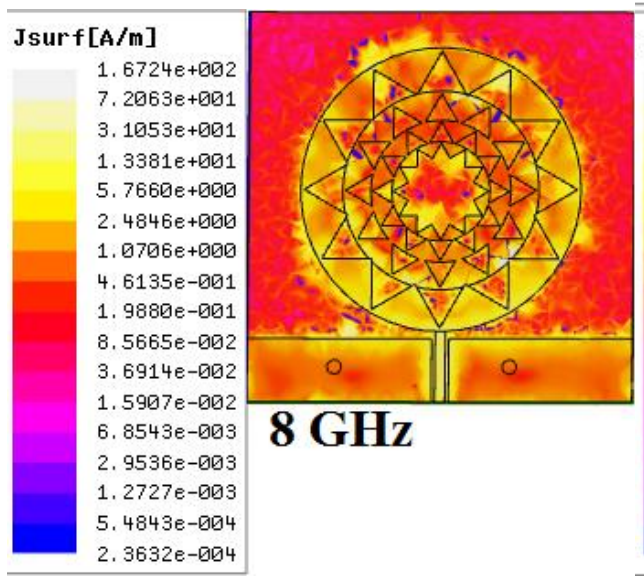

iii.

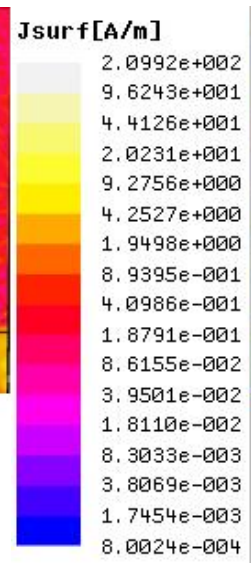

1.7.

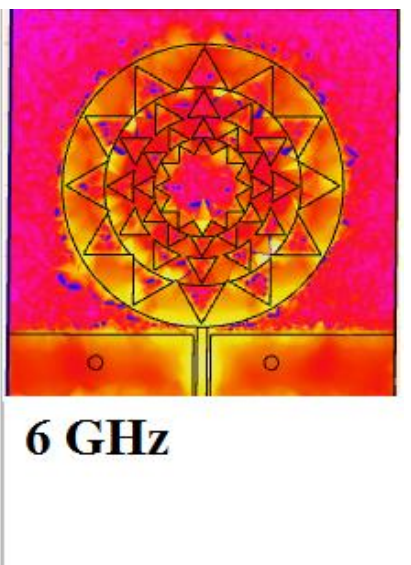

ii.
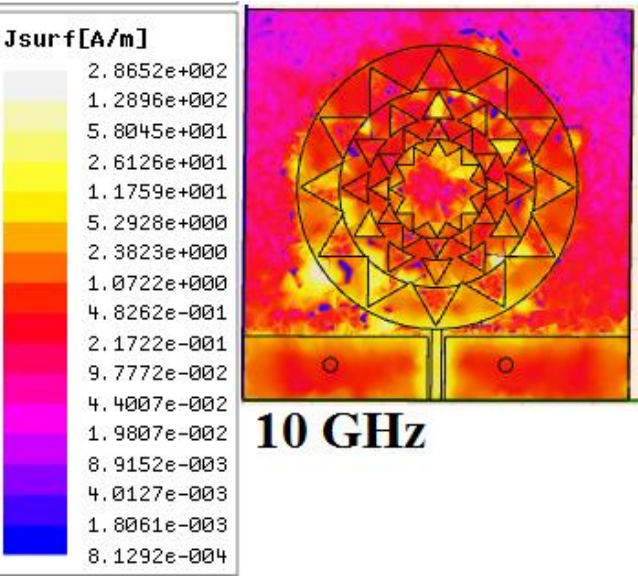

iv.

Figure 13. Surface current distribution $\left(\mathbf{J}_{\text {surf }}\right)$ pattern of planar monopole.

and 90 deg. in theta plane as seen in Figure11(iii) and Figure11(iv). The maximum gain of 9 $\mathrm{dB}$ has been obtained around $10 \mathrm{GHz}$. These radiating characteristics are suitable for microwave UWB systems [15], [26].

The distortions in the patterns might be attributed to fractal shape of the monopole, reflections from metallic surfaces and edge diffraction and excitations of higher order modes [23]-[25].

\section{Conclusions}

Printed monopole antenna designed on low cost FR-4 substrate material in planar and vertical configurations using fractal patch and CPW feed has been investigated. In planar monopole antenna the feed of the antenna, ground and radiating elements all are printed on the same side of the substrate without any via (plated through hole) for ground purpose. So, this technique is less costly. But, vertical monopole antenna due to its ground becomes larger in volume. The printed fractal monopole antenna offers ultra and super wideband characteristics respectively in planar and vertical configurations due to coupled resonating effect of closely associated band of frequencies when fed with $\mathrm{CPW}$ feed technique. The monopole antenna offers maximum gain of $9 \mathrm{~dB}$ in theta plane and $3 \mathrm{~dB}$ in phi plane at observed frequencies. The antenna is useful for UWB and SWB applications. The self similar current distribution in the antenna can be linked to 
International Journal Of Microwave Engineering (JMICRO) Vol.1, No.4, October 2016 self-similar radiation characteristics. The antenna exhibits nearly omni-directional radiation characteristics in azimuth plane at all frequencies.

\section{ACKNOWLEDGEMENTS}

The authors would like to thank everyone, just everyone!

\section{REFERENCES}

[1] Kumar and K. P. Ray, Broadband Microstrip Antennas, Norwood, Mass.: Artech House, 2003.

[2] K. L. Wong, Compact Broadband Microstrip Antennas, New York: John Wiley \& Sons, Inc., 2002.

[3] M. G. Sindou, Ablart, and C. Sourdois, "Multiband and wideband properties of printed fractal branched antennas," Electron. Lett., vol. 35, pp. 181-182, Feb. 1999.

[4] L. Shafai, Antenna Engineering Handbook, $4^{\text {th }}$ ed. New York: McGraw-Hill, 2007, pp. 16-49, Jun. 2008,

[5] T. Y. Han et al., "Unidirectional circularly-polarized slot antennas with broadband operation," IEEE Trans. Antennas Propog., vol. 56, pp. 1780-1783, Jun. 2008.

[6] Hara Prasad etal., "Microstrip fractal patch antenna for multiband communication," Electronic Lett., vol. 36, issue 14, pp. 1179-1180, July 2000.

[7] A. N. Wang, and W. X. Zhang, "Design and optimization of broadband circularly polarized wideslot antenna," Journal of Electromagnetic Waves and Applications, vol. 23, no. 16, pp. 2229 2236, 2009.

[8] R. Joseph, S. Nakao, and T. Fukusako, "Circular slot antennas using 1-shaped probe for broadband circular polarization," Progress in Electromagnetics Research C, vol. 18, pp. 153-168, 2011.

[9] Sze, and Wong, "Bandwidth enhancement of a microstrip-line-fed printed wide-slot antenna," IEEE Trans. Antennas and Propog., vol. 49, no. 7, pp.1020 - 1024, Jul. 2001.

[10] X. H. Yang, and L. Shafai, "Characteristics of aperture-coupled microstrip antennas with various radiating patches and coupling slots," IEEE Trans. Antennas Propag., vol. 43, no. 1, pp. 72-78, Jan.1995.

[11] Thakare, Y. B. etal., "Super wideband fractal antenna for wireless communication," in Conf. Rec. 2012 IEEE Int. Conf. ICWITS, pp.1-4, Nov.2012.doi: 10.1109/ICWITS.2012.6417706.

[12] Y. B. Thakare et al., "Design of fractal patch antenna for size and radar cross-section reduction," IET Microw. Antennas and Propog., vol. 4, pp. 176-178, 2010.

[13] D. H. Werner, and P. L. Werner, "Frequency independent features of self-similar fractal antennas," Radio science, vol. 31, no. 6, pp.1331-1343,Nov.-Dec. 1996.

[14] C. Puente et al., "Small but long koch fractal monopole," Electron. Lett. , vol. 34, issue 1, pp. 910, Jan. 1998.

[15] G. J. Walker, and J. R. James, "Fractal volume antennas," Electron. Lett., vol. 34, issue 16, pp. 1536-1537, Aug. 1998.

[16] Y. B. Thakare, "Design of fractal antennas and their back scattering analysis," Ph.D. dissertation, Dept. Electron. Sci., Shivaji Univ., Kolhapur, India, 2012.

[17] N. P. Agrawal, "Wideband planar monopole antennas," IEEE Trans. Antennas and Propag., vol. 46, no. 2, pp. $294-295$, Feb. 1998.

[18] L. Jianxin et al., "Study of a printed circular disc monopole antenna for UWB systems," IEEE Trans. Antennas Propag., vol. 53, issue11, pp. 3500 - 3504, Nov. 2005.

[19] X. H. Wu, and A. A. Kishk, "Study of an ultra wideband omni directional rolled monopole antenna with trapezoidal cuts," IEEE Trans. Antennas Propag., vol. 56, issue 1, pp. 259-263, Jan. 2008.

[20] Anuj Batra etal., "Design of a Multiband OFDM System for Realistic UWB Channel Environments," IEEE Trans. Microw. Theory Tech., vol. 52, no. 9, pp. 2123-2138, Sept. 2004.

[21] Chi-Chih Chen, Antenna Engineering Handbook, $4^{\text {th }}$ ed. New York: McGraw-Hill, pp. 16-49, 2007. 
International Journal Of Microwave Engineering (JMICRO) Vol.1, No.4, October 2016

[22] S. A. Evangeolos, et al., "Circular and elliptical CPW fed slot and microstrip fed antennas for ultra - wideband applications," IEEE Antennas and Wireless Propag. Lett., vol. 5, issue 1, pp. 294-297 Dec. 2006.

[23] K. P. Ray, "Design aspects of printed monopole antennas for ultra-wideband applications", Hindawi Corporation International Publishing Journal of Antennas and Propagation, 2008.

[24] K. Francis Jacob, "Printed monopole antenna for ultra wide band (UWB) applications - A Thesis in Microwave Electronics," Cochin University of Science and Technology, Cochin-22, India, June 2008

[25] Cheng-Nan Hu, and Ching-Kuang C. Tzuang, "Analysis and design of large leaky-mode array employing the coupled-mode approach," IEEE Microw. Theory Tech., vol. 49, no. 4, April 2001, pp. 629-636.

[26] M. Navarro et al., "Self-similar surface current distribution on fractal Sierpinski antenna verified with infrared thermograms," in Conf. Rec. IEEE APS Int. Symp. , vol.3, pp. 1566-1569, 1999.

\section{Authors}

YOGESH BABARAOJI THAKARE

Short Biography

Yogesh B. Thakare (mother-Sushilabai) is passed BE E\&TC in the year 1998 with first class from VYWS College of Engg., Badnera, Amravati University (Maharashtra -India), ME E\&TC (Microwave) in the year 2006 from Govt. College of Engg., Pune, Pune University (Maharashtra-India) in first class and Ph.D. (Electronics) from Shivaji University, Kolhapur (Maharashtra- India) in 2015. He has about 15 years teaching and 02 years industrial experience. He is currently working with PVG's College of Engineering and Technology, Parvati, Pune (Maharashtra-India) as ASSISTANT PROFESSOR in the department of electronics and telecommunication Engg. He taught major sub ject like wave theory and antenna, microwave engineering, semiconductor devices and technology, analogue and digital communication engineering. He has 04 international and 01 national journal and 05 international and 04 national conference publications. His current research interest is in wave propagation techniques, microstrip antenna engineering, communication engineering and semiconductor electronics. 